\title{
Development of a method for environmentally friendly chemical peptide synthesis in water using water-dispersible amino acid nanoparticles
}

\author{
Keiko Hojo*, Asaki Hara, Hiroyuki Kitai, Mare Onishi, Hideki Ichikawa, Yoshinobu Fukumori and Koichi Kawasaki
}

\begin{abstract}
Due to the vast importance of peptides in biological processes, there is an escalating need for synthetic peptides to be used in a wide variety of applications. However, the consumption of organic solvent is extremely large in chemical peptide syntheses because of the multiple condensation steps in organic solvents. That is, the current synthesis method is not environmentally friendly. From the viewpoint of green sustainable chemistry, we focused on developing an organic solvent-free synthetic method using water, an environmentally friendly solvent. Here we described in-water synthesis technology using water-dispersible protected amino acids.
\end{abstract}

\section{Introduction}

In current industrial processes, the synthesis of organic compounds usually by dissolving material compounds in organic solvents is widely used. More stringent rules regulating the use and disposal of organic solvents are increasingly restricting chemical synthesis alternatives, and there is an urgent need to develop non-hazardous alternatives in order to achieve green sustainable chemistry [1,2]. Reduction of the use of harmful organic solvents is required to clean the chemical synthesis processes, reduce waste, and ensure safety.

As a result of major advances in molecular biology and medicine, the importance of the peptides in biological processes has been attracting attention, and the need to accelerate the supply of peptide by chemical synthesis is rapidly increasing. However, a great deal of organic solvent is consumed in chemical peptide syntheses because of the multiple condensation steps in organic solvents. With their E-factors [3], a useful measure of the potential environmental acceptability of chemical processes, that are estimated to be 100 times larger than that of normal synthetic compounds, chemical peptide syntheses are almost incomplete in terms of environmental friendliness. Therefore, development of a method for organic solvent-free peptide synthesis is strongly desired. If water

\footnotetext{
* Correspondence: hojo@pharm.kobegakuin.ac.jp

Faculty of Pharmaceutical Sciences \& Cooperative Research Center of Life Sciences, Kobe Gakuin University, Chuo-ku, Kobe 650-8586, Japan
}

is substituted for organic solvents, the use of organic solvents can be reduced radically. In conventional organic synthesis, it is common to dissolve reactant molecules in solvents to carry out the reaction efficiently. Peptide synthesis can be carried out in water via chemical conversion of protected amino acids to water-soluble forms [4-7]. In this case, however, additional conversion steps are required in total synthetic process, and it is not desirable in terms of preparation costs, resource saving, and energy conservation. Thus, development of simplified techniques other than chemical conversion is urgently needed for environmentally friendly peptide synthesis.

Chemical peptide synthesis is classified into two types: solid-phase synthesis and solution-phase synthesis. Choosing the best amino acid derivatives is one of the most important, and sometimes difficult, aspects of peptide synthesis. Currently, Fmoc-amino acids are routinely used building blocks for solid-phase peptide synthesis $[8,9]$. In solution-phase synthesis, tert-butyloxycarbonyl (Boc)-protected amino acids are commonly used. Both Fmoc- and Boc-amino acids are highly soluble in ordinary organic solvents. These molecules are sparingly soluble in water and are considered inappropriate for in-water peptide synthesis. We have developed technology using these common water-insoluble protected amino acids processed into water-dispersible nanoparticles to allow synthesis in water, and have reported that the use of water-dispersible nanoparticles is effective in dramatically reducing reaction time in water [10-12]. 
This review describes a method for solid-phase and solution-phase peptide synthesis in-water using water-dispersible protected amino acid nanoparticles.

\section{Development of solid-phase peptide synthesis in water using Fmoc-protected amino acids}

The solid-phase concept, in which the reaction is carried out on a solid support, was originally developed by Merrifield [13]. Solid-phase synthesis typically offers many advantages over the conventional synthesis in terms of efficiency as well as due to the convenient applicability and purification procedures. Since solidphase synthesis is performed in a two-phase (liquid and solid phases) medium, for efficient solid-phase reaction, it is necessary that therefore the building blocks and reagents generally have good solubility in solvents. Currently, solid-phase peptide synthesis is performed by the Fmoc strategy in most cases. Fmoc-protected amino acids, however, are poorly soluble in water and therefore not adequate for use in the solid-phase synthesis in water. Recently with the aim of achieving solid-phase peptide synthesis in water, we developed in-water solid phase synthesis using water-dispersible nanoparticulate Fmoc-amino acids (Figure 1) [10,11].

In recent years, utilization of nanoparticle-based technology has emerged as a strategy to tackle formulation problems associated with poorly water-soluble drugs [14-16]. Grinding particles to nanosize can lead to increased specific surface area and homogenous mixing of multiple components. We therefore predicted that conversion of Fmoc-amino acids into homogenously water-dispersible nanoparticles would enable increased specific surface area and homogenous mixing with the resin in water, which might lead to smooth progress of the solid phase reaction in water according to the Fmoc approach (Figure 2).

Nanoparticles can be produced by dispersion-based processes. Wet milling is an attrition-based process in which the insoluble material is dispersed in the aqueousbased surfactant solution, and the resulting material is

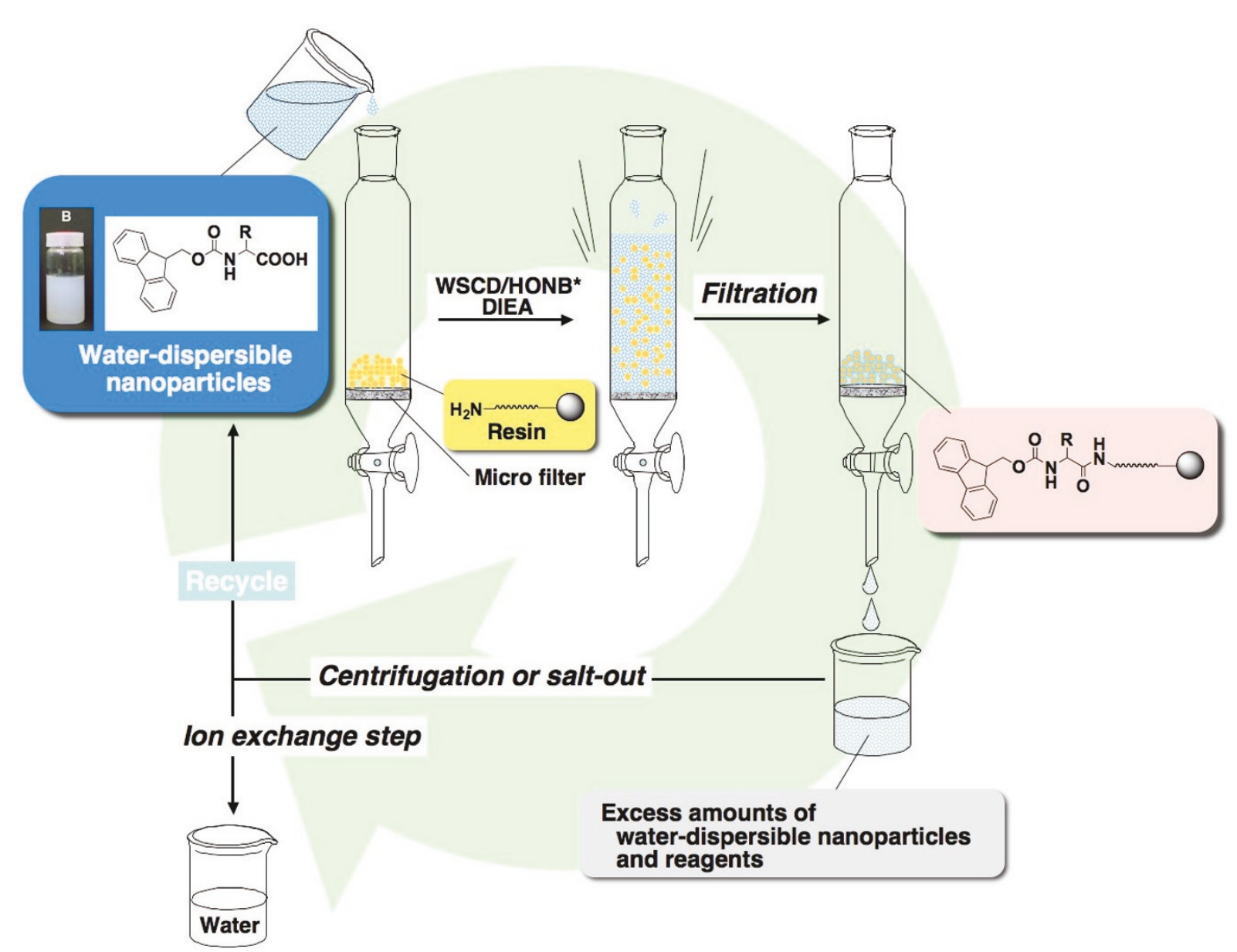

Figure 1 The solid-phase peptide synthesis in water using water-dispersible Fmoc-amino acid nanoparticles. 

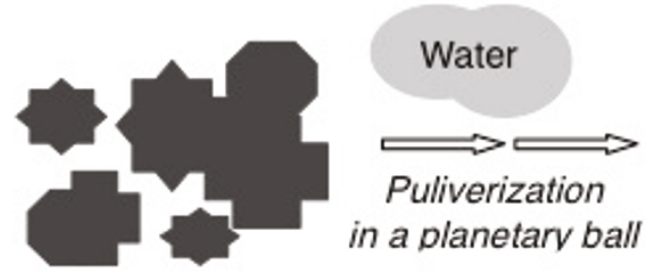

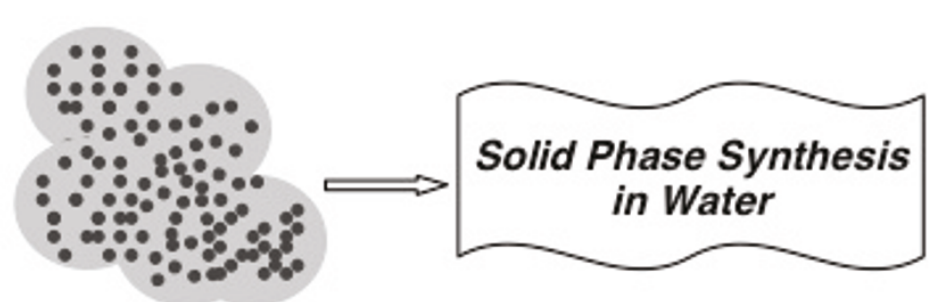

\section{Unprocessed Fmoc-amino acid Water-dispersible nanoparticles}

Figure 2 Nanosaizing leads to smooth progress of the solid phase reaction in water.

converted into water-dispersible nanoparticles. Waterdispersible nanoparticles of Fmoc-amino acids were prepared by wet milling using planetary ball mills in the presence of PEG as a dispersion agent (Figure 3) [10]. The grinding media was composed of zirconium oxide beads. The average sizes of the resulting water-dispersible nanoparticles were determined by dynamic light scattering (DLS) analysis, and were found to be between 250 $500 \mathrm{~nm}$. In Figure 4, photo images of the Fmoc-Phe-OH in water are shown before (Figure 4(A)) and after conversion into water-dispersible nanoparticles (Figure 4(B)). Scanning electron microscopy (SEM) images of the same nanoparticles also revealed formation of Fmoc-Phe-OH nano-sized particles (Figure 5).

It was hypothesized that decreasing the Fmoc-amino acid particle size to nano-scale could improve the efficiency of the in-water solid-phase reaction. Therefore, we next examined in-water solid-phase reaction using the water-dispersible nanoparticles [10]. Table 1 summarizes the results of the in-water solid-phase coupling reaction of the water-dispersible Fmoc-Phe-OH nanoparticles with the H-Leu-Rink amide-TentaGel resin using water-soluble coupling reagents, water-soluble carbodiimide (WSCI, 1-ethyl-3-(3'-dimethylaminopropyl)carbodiimide

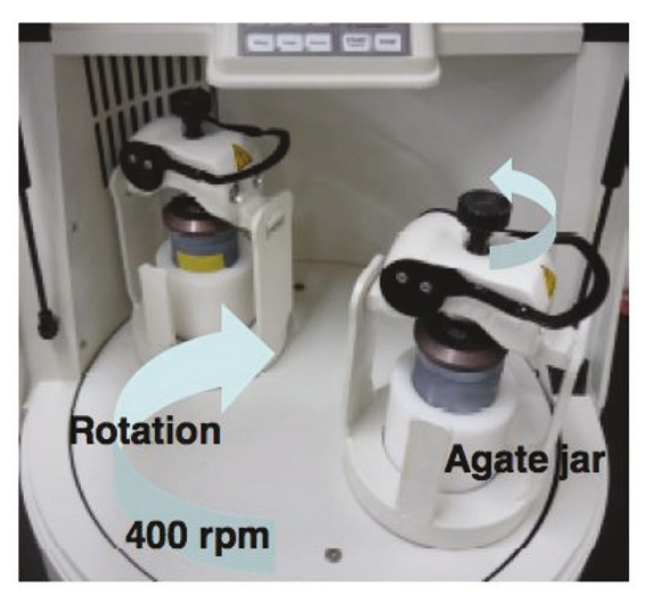

Figure 3 Wet-milling in planetary ball-mill. hydrochloride) [17] in combination with $N$-hydroxy-5norbornene-endo-2,3-dicarboximide (HONB) [18] and $N$, $\mathrm{N}$-diisopropylethylamine (DIEA). The coupling rate was $68 \%$ after 15 min of reaction in water (entry 1 ), and the rate became nearly quantitative after $30 \mathrm{~min}$ (entry 2). Coupling reactions using water-dispersible nanoparticles were found to proceed quantitatively in approximately $30 \mathrm{~min}$, even on the solid-phase in water. These reactions were smoother than expected, suggesting that the coupling reaction between an aqueous nanocolloid and a solid support could be completed in a short time period. This high coupling reaction efficiency might be attributed to the homogenous mixing of the nanoparticles with a resin in water.

To establish a peptide synthesis method using water-dispersible Fmoc-amino acid nanoparticles, we tried to synthesize the 5-opioid peptides, Leu-enkephalinamide (Tyr-Gly-Gly-Phe-Leu- $\mathrm{NH}_{2}$ ) [10]. Table 2 shows the synthetic protocol in which all steps are carried out in aqueous media. Rink amide-TentaGel resin was used as a support for solid-phase synthesis, and WSCI, HONB, and DIEA were used for condensation reactions. Figure 6(C) shows the HPLC analysis profile of the Leu-enkephalinamide synthesized using the water-dispersible Fmoc-amino

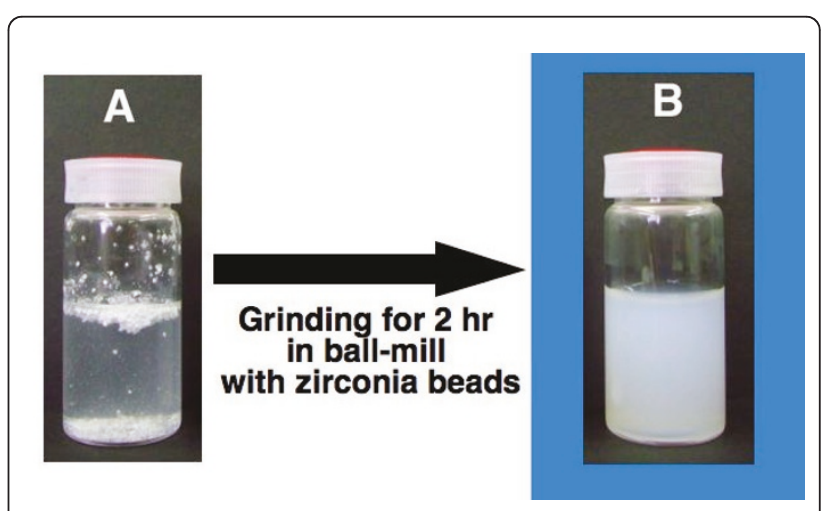

Figure 4 Photo image of Fmoc-Phe-OH particles. (A) unprocessed Fmoc-Phe-OH particles; (B) water-dispersible FmocPhe-OH nanoparticles. 

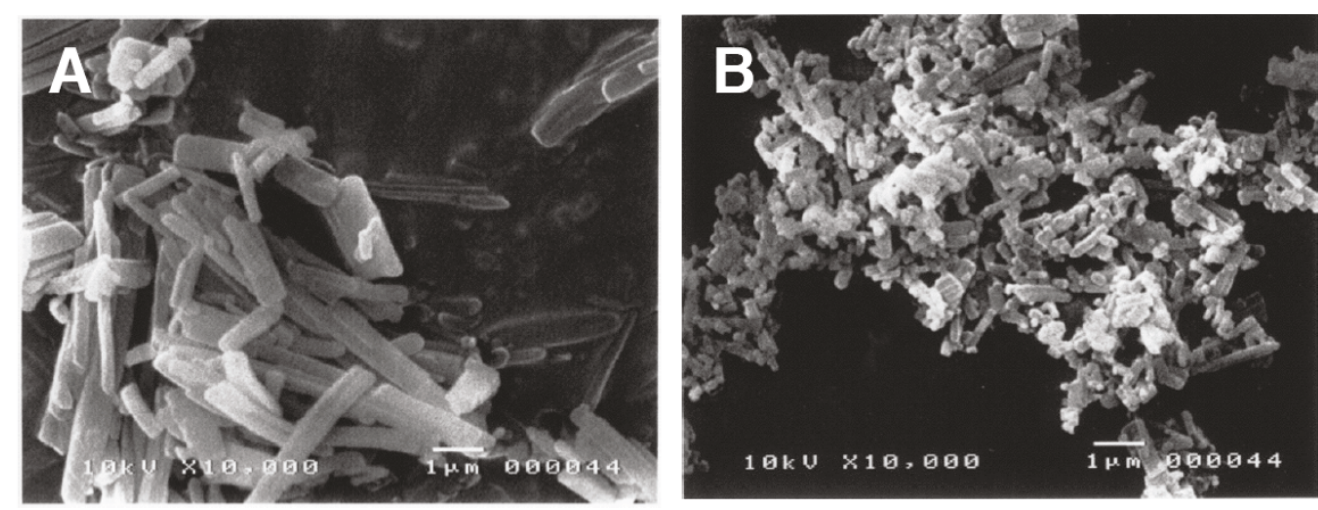

Figure 5 SEM image of Fmoc-Phe-OH particles. (A) unprocessed Fmoc-Phe-OH particles; (B) water-dispersible Fmoc-Phe-OH nanoparticles.

acid nanoparticles. Clearly, the data shows a main peak with $67 \%$ yield. We also attempted to synthesize Leuenkephalinamide by solid-phase synthesis using the unprocessed Fmoc-amino acids in water and in aqueous PEG solution, and the HPLC analysis profiles of the resultant products are shown in Figure 6(A) and Figure 6(B), respectively. As shown, in both cases no peak was seen with the retention time for Leu-enkephalinamide. These results suggest that the in-water synthesis using the unprocessed Fmoc-amino acids is unachievable, even in the presence of dispersible PEG.

One of the important factors for good solid-phase reactivity in water is the homogeneous mixing of the nanoparticulate amino acids and a resin. Since active surfactants are known as excellent dispersible agents, nanosuspensions of protected amino acids dispersed with active surfactants would lead to further good reactivity even in water. Triton X-100 is known to inhibit aggregation of the peptide chain. We examined preparation of dispersible Fmoc-amino acid nanoparticles in water containing Triton X-100 as a dispersion agent. The sizes of the resulting water-dispersible nanoparticles were summarized in Table 3 . The best condition is entry 2 in which nanoparticles were prepared in aqueous $0.2 \%$ Triton $\mathrm{X}-100$ solution using $0.5 \mathrm{~mm}$ zirconium oxide beads. The minimum average particle size observed was $217 \mathrm{~nm}$. It was not possible to reduce the sizes of all particles to $100 \mathrm{~nm}$ or less. Next we synthesized Leu-

Table $\mathbf{1}$ In-water solid-phase coupling reaction using
water-dispersible nanoparticles ${ }^{\boldsymbol{a}}$
\begin{tabular}{llllll}
\hline Entry & Reagent & Additive & Base & Time [min] & Yield [\%] $^{\boldsymbol{b}}$ \\
\hline 1 & WSCl & HONB & DIEA & 15 & 68 \\
2 & WSCl & HONB & DIEA & 30 & 100
\end{tabular}

${ }^{a}$ Reactions were carried out between water-dispersible Fmoc-Phe-OH nanoparticles and $\mathrm{H}$-Leu-Rink amide TentaGel resin. ${ }^{b}$ After reaction the resin was hydrized and Phe and Leu content in hydrolysates were analyzed. The yield of coupling reaction was calculated from the amino acid ration of Phe and Leu by amino acid analysis. enkephalinamide by in-water solid-phase method described in Table 2 using dispersible Fmoc-amino acid nanoparticles in water containing Triton X-100 [11]. HPLC analysis showed a single major peak corresponding to Leu-enkephalinamide, and the total yield was $79 \%$. The purities of crude peptides cleaved from the resin were over $90 \%$ and similar to the results by the general Fmoc synthesis method using organic solvents.

Thus, to summarize our results, we specifically developed efficient in-water reaction using water sparingly soluble protected amino acids in which dispersed in water, and verified their utilities by successful solidphase synthesis of Leu-enkephalinamide in water. General solid-phase syntheses are based on reactions of solids and liquids, while this method is based on reactions between a solid reactant and an aqueous nanocolloidal reactant. These results suggest that our method can be applied to every organic reaction. There are some advantages of using the water-dispersed nanoparticulate reactants over the water-soluble reactants. In the case of in-water synthesis using water-soluble protected amino acids, after the in-water reaction the excess reactants remain dissolved in water, causing possibilities of water contamination. In contrast, after in-water reaction using nanoparticles, the nanoparticles could be easily separated from the resin by filtering through a microfilter (Figure 1). The particles in the filtrate could also be

Table 2 Protocol for in-water, solid-phase synthesis using nanoparticles

\begin{tabular}{lll}
\hline Step & Reagent & Time \\
\hline 1 & Water & $1 \mathrm{~min} \times 5$ \\
2 & Water-dispersible Fmoc-amino acid nanoparticle & $1 \mathrm{hr}$ \\
& WSCD, HONB, DIEA & \\
3 & Water & $1 \mathrm{~min} \times 5$ \\
4 & Aqueous 50\% EtOH & $1 \mathrm{~min} \times 2$ \\
5 & $0.1 \mathrm{~N} \mathrm{NaOH}$ aqueous $/ 90 \% \mathrm{EtOH}$ & $5 \mathrm{~min} \times 3$ \\
6 & Aqueous $50 \% \mathrm{EtOH}$ & $1 \mathrm{~min} \times 2$ \\
\hline
\end{tabular}




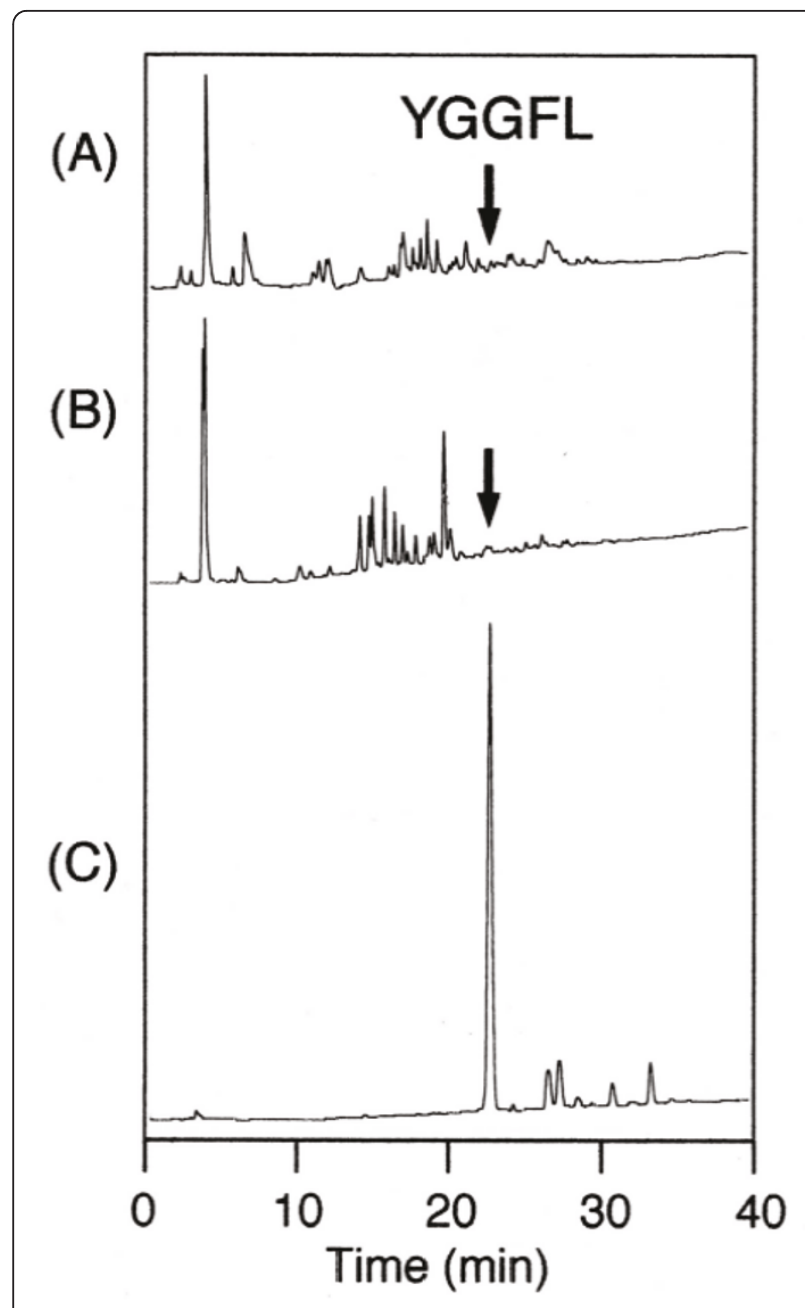

Figure 6 HPLC analysis data of Leu-enkephalinamide after cleavage from the resin. The peptide was prepared by in-water, solid-phase synthesis using (A) unprocessed Fmoc-amino acids, (B) unprocessed Fmoc-amino acids with PEG, and (C) water-dispersible nanoparticulate Fmoc-amino acids. Elution was carried out for over $40 \mathrm{~min}$ at a flow rate of $1 \mathrm{ml} / \mathrm{min}$ with a linear gradient from 9:1 to 5:5 mixture of $0.05 \%$ aqueous TFA and $0.05 \%$ TFA in acetonitrile.

Table 3 Grinding condition and particle size of waterdispersible Fmoc-Ala-OH nanoparticles ${ }^{a}$

\begin{tabular}{|c|c|c|c|}
\hline Entry & Detargent agent & $\begin{array}{l}\text { Bead Size } \\
{[\mathrm{mm}]}\end{array}$ & $\begin{array}{l}\text { Particle Size } \\
\text { [nm] }\end{array}$ \\
\hline 1 & $0.4 \%$ Triton $\mathrm{X}-100$ solution & 0.5 & $292 \pm 119$ \\
\hline 2 & $0.2 \%$ Triton X-100 solution & 0.5 & $217 \pm 89$ \\
\hline 3 & $0.2 \%$ Triton X-100 solution & 1.0 & $362 \pm 157$ \\
\hline 4 & $0.1 \%$ Triton $\mathrm{X}-100$ solution & 1.0 & $484 \pm 225$ \\
\hline 5 & $\begin{array}{l}0.01 \% \text { Triton X-100 } \\
\text { solution }\end{array}$ & 1.0 & $2581 \pm 954$ \\
\hline
\end{tabular}

${ }^{a}$ An aqueous dispersion of nanoparticulate $\mathrm{Fmoc}-\mathrm{Ala}-\mathrm{OH}$ was prepares by grinding using a planetary ball mill. An agate jar was charged with precleaned zirconium oxide beads, Fmoc-Ala-OH, and detergent solution. The batch was rolled for $3 \mathrm{hr}$. After grinding, the beads were removed by filtration with water. easily separated from the water by centrifugation or by using salting-out technique. Thus, the nanoparticlebased technology described here leads a major step forward in the development of environmentally friendly solid-phase synthesis method.

\section{Development of solution-phase peptide synthesis in} water using water-dispersible submicron particles of Bocprotected amino acids

Industrial synthesis of peptides on the market, including pharmaceutical products, is generally performed in organic solvents by the solution-phase. Boc-amino acid is widely used in the industrial chemistry and wellknown to be suitable for green chemistry because only gases were generated in the removal step of Boc group. Although Boc-amino acids are highly soluble in organic solvent, these are sparingly soluble in water. Therefore, we also developed a method to synthesize peptides in water by the solution-phase using water-dispersible Boc-amino acid nanoparticles [12]. When Boc-amino acids are converted to nanoparticles homogenously dispersed in water, the specific surface area is increased and water dissolution speed is improved. We hypothesized that the reaction can be dramatically accelated, even when water-insoluble Boc amino acids are used (Figure 7).

First, water-dispersible nanoparticles of Boc-protected amino acid were prepared by the method of wet grinding using planetary ball mills [12]. In the presence of PEG as a dispersant, Boc-amino acid was ground with zirconia beads in water using a planetary ball mill for 2 hours at room temperature. The beads were then removed by filtration and water-dispersible nanoparticles of Boc-amino acid were obtained. The sizes of the resulting waterdispersible nanoparticles were determined by DLS analysis, and were found to be between $500 \sim 750 \mathrm{~nm}$. The obtained nanoparticles showed the Tyndall phenomenon upon laser irradiation and readily passed through a filter paper (ADVANTEC Filter Paper No.2) (Figure 8).

Next, we examined in-water coupling reactions using the prepared water-dispersible nanoparticles of Boc-amino acid [12]. For in-water reaction, coupling reagents must be soluble in water and their reactivity must be maintained. Most commercially available water-soluble coupling reagents are developed focusing on removability by water after a reaction. The water-soluble coupling reagents, WSCI and 4-(4,6-dimethoxy-1,3,5-triazin-2-yl)-4-methylmorpholinium chloride (DMTMM) $[19,20]$, were tested in the coupling reaction between Boc-Phe-OH nanoparticles and $\mathrm{H}$-Leu- $\mathrm{NH}_{2}$. These results were summarized in Table 4. Clearly, results using the WSCI combination containing the additives HONB, $\mathrm{N}$-hydroxysuccinimide (HOSu) $[21,22]$ or 3 -sulfo- $N$-hydroxysuccinimide (sulfo-HOSu) [23] were not satisfactory (entries 2-5). The in-water 


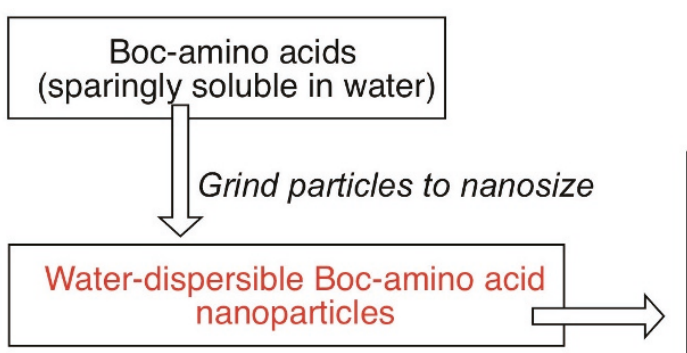

\section{Smooth progress of the reaction in water}

1. Increased specific surface area

2. Homogenous mixing with reagents in water

3. Improvement of dissolution speed in water

Figure 7 New physical properties and reactivities acquired by water-dispersible nanoparticulation

a)

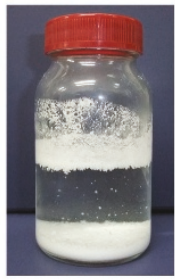

b)

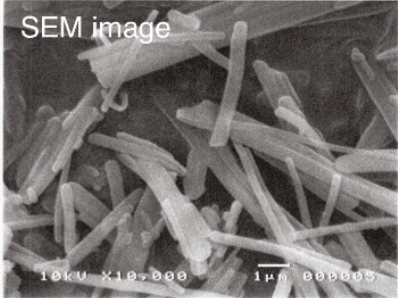

C)

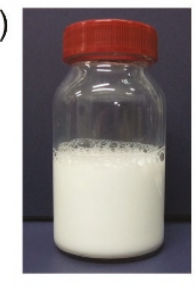

d)

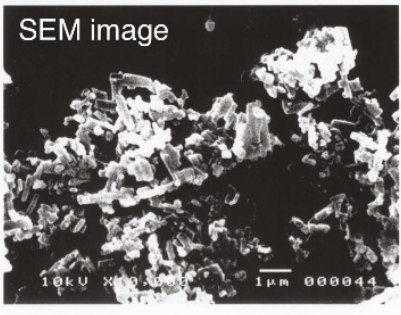

e)

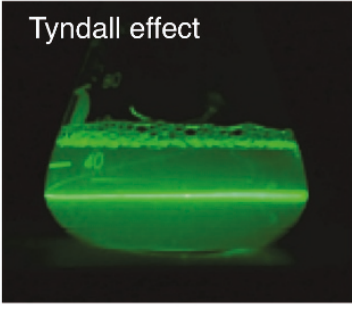

f)

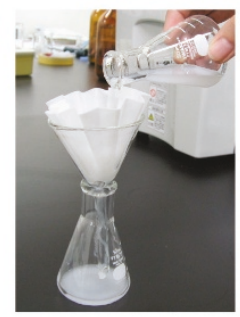

Figure 8 Boc-amino acids are converted to nanoparticles homogenously dispersed in water. a) A photo image of unprocessed Boc-Phe$\mathrm{OH}$. b) A SEM imagea of unprocessed Boc-Phe-OH. c) A photo image of water-dispersible nanoparticle Boc-Phe-OH. d) SEM image of waterdispersible nanoparticle Boc-Phe-OH. e) Water-dispersible Boc-Phe-OH nanoparticles showed the Tyndall phenomenon upon laser irradiation. $f$ ) Water-dispersible nanoparticle Boc-Phe-OH readily passed through a filter paper.

Table 4 In-water coupling reaction using water-dispersible nanoparticles ${ }^{a}$

\begin{tabular}{|c|c|c|c|c|}
\hline Entry & Reagent & Additive & Base & Yield [\%] $^{b}$ \\
\hline 1 & DMTMM & - & NMM & 89 \\
\hline 2 & WSCl & HONB & DIEA & 25 \\
\hline 3 & WSCl & HOSu & DIEA & 32 \\
\hline 4 & WSCI & Sulfo-HOSu & DIEA & 71 \\
\hline
\end{tabular}

${ }^{a}$ Reactions were carried out between $\mathrm{H}$-Leu- $\mathrm{NH}_{2}$ and water-dispersible Boc-Phe-OH nanoparticles. ${ }^{b}$ The yield of peptides was calculated from analytical HPLC profiles.

coupling reaction using DMTMM in the presence of NMM resulted in good yields (89\%).

To evaluate water-dispersible Boc-amino acid nanoparticles, we attempted to synthesize Leu-enkephalinamide by in-water solid-phase synthesis using water-dispersible nanoparticles (Figure 9) [12]. Coupling reactions were carried out by mixing the water-dispersible nanoparticles of Boc-protected amino acid and amine component in water using DMTMM and then stirring the mixture under neutral conditions for 12 hours at room temperature (Figure 10). Since nanoparticles readily pass through a micro-pore filter and the reagent is water-soluble, post-coupling treatment consists only of removing the unreacted nanoparticles and reagent-mediated byproducts by filtration to obtain the product precipitates. Almost all of the protected peptide fragments showed a single peak without any special purification steps other than filtration (Figure 11). In any case, peptide was 


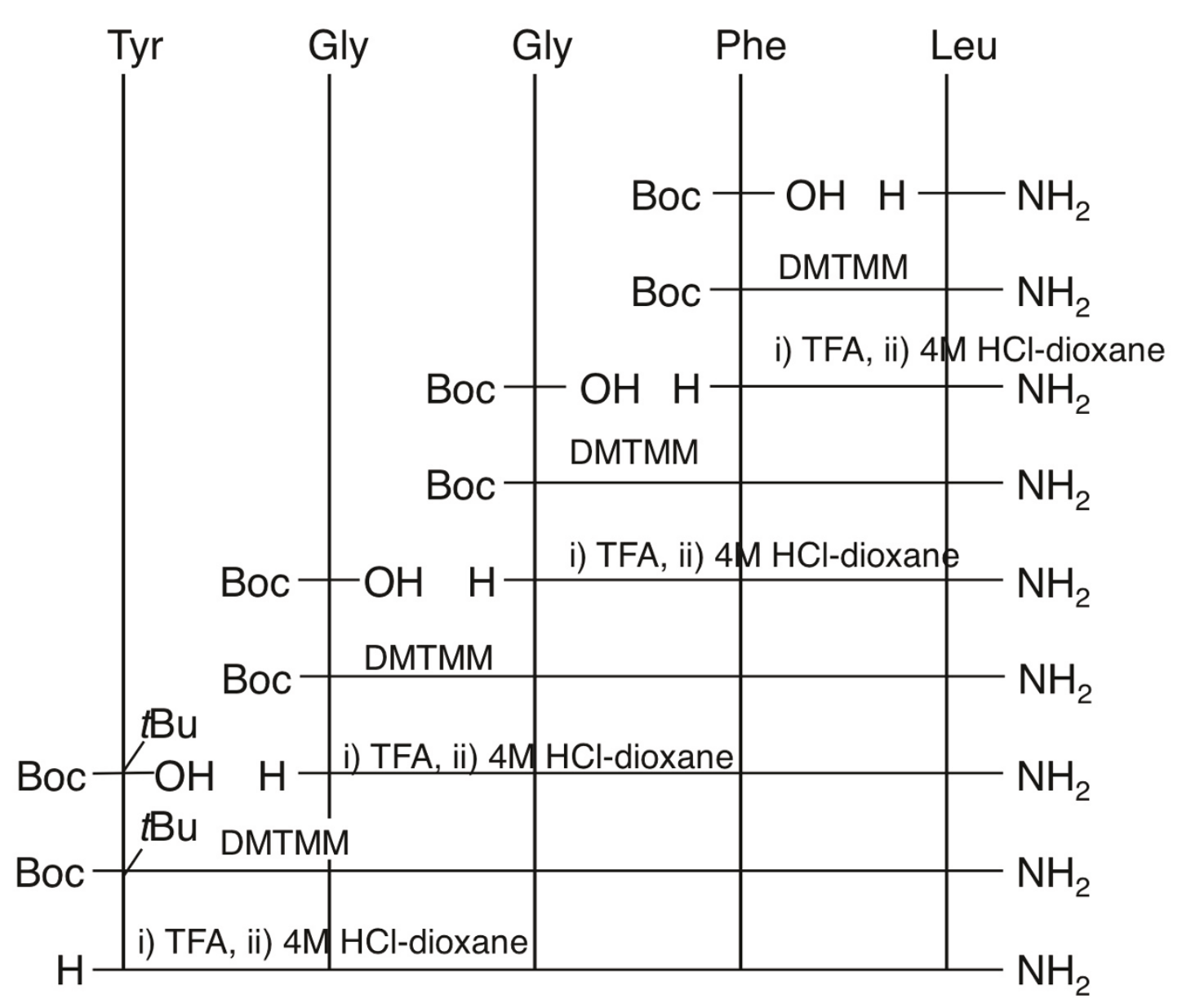

Figure 9 Synthetic scheme of Leu-enkephalinamide

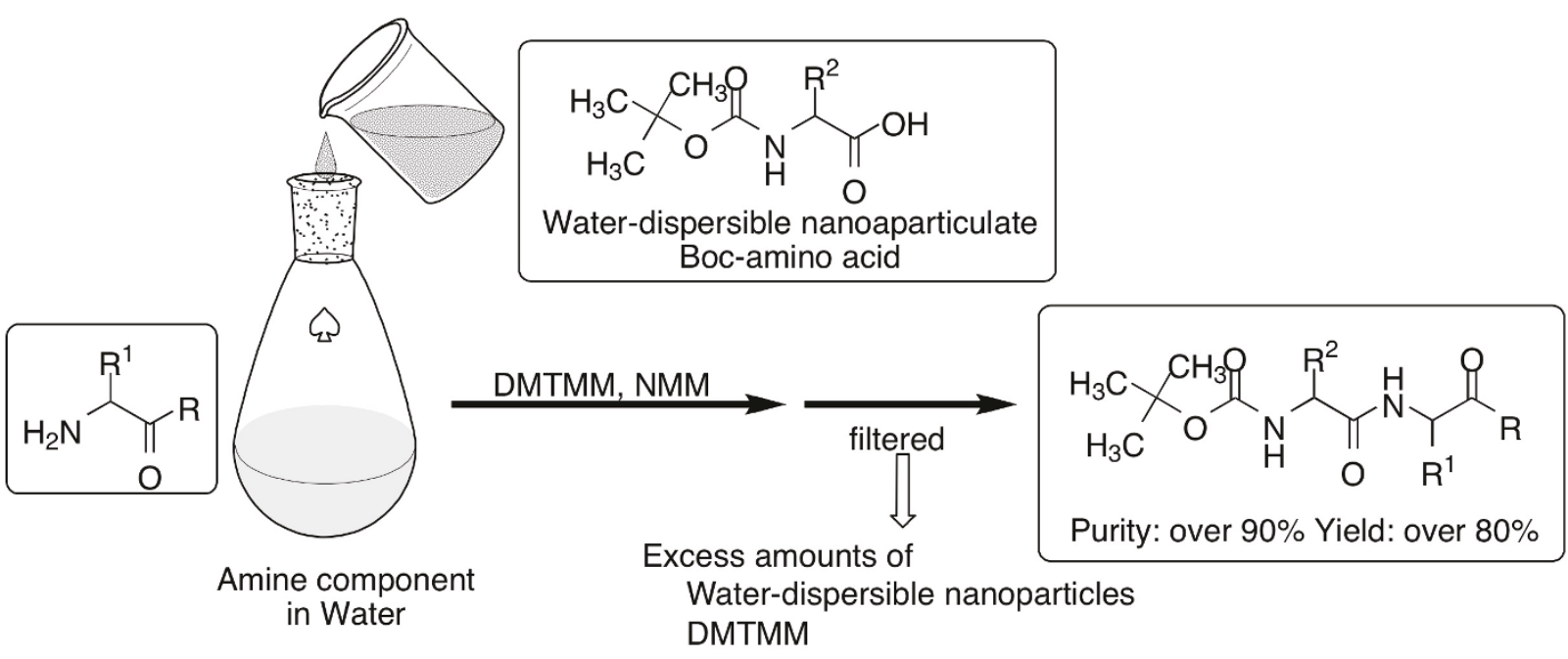

Figure 10 In-water coupling reaction using a water-dispersible nanoparticle Boc-amino acid. 

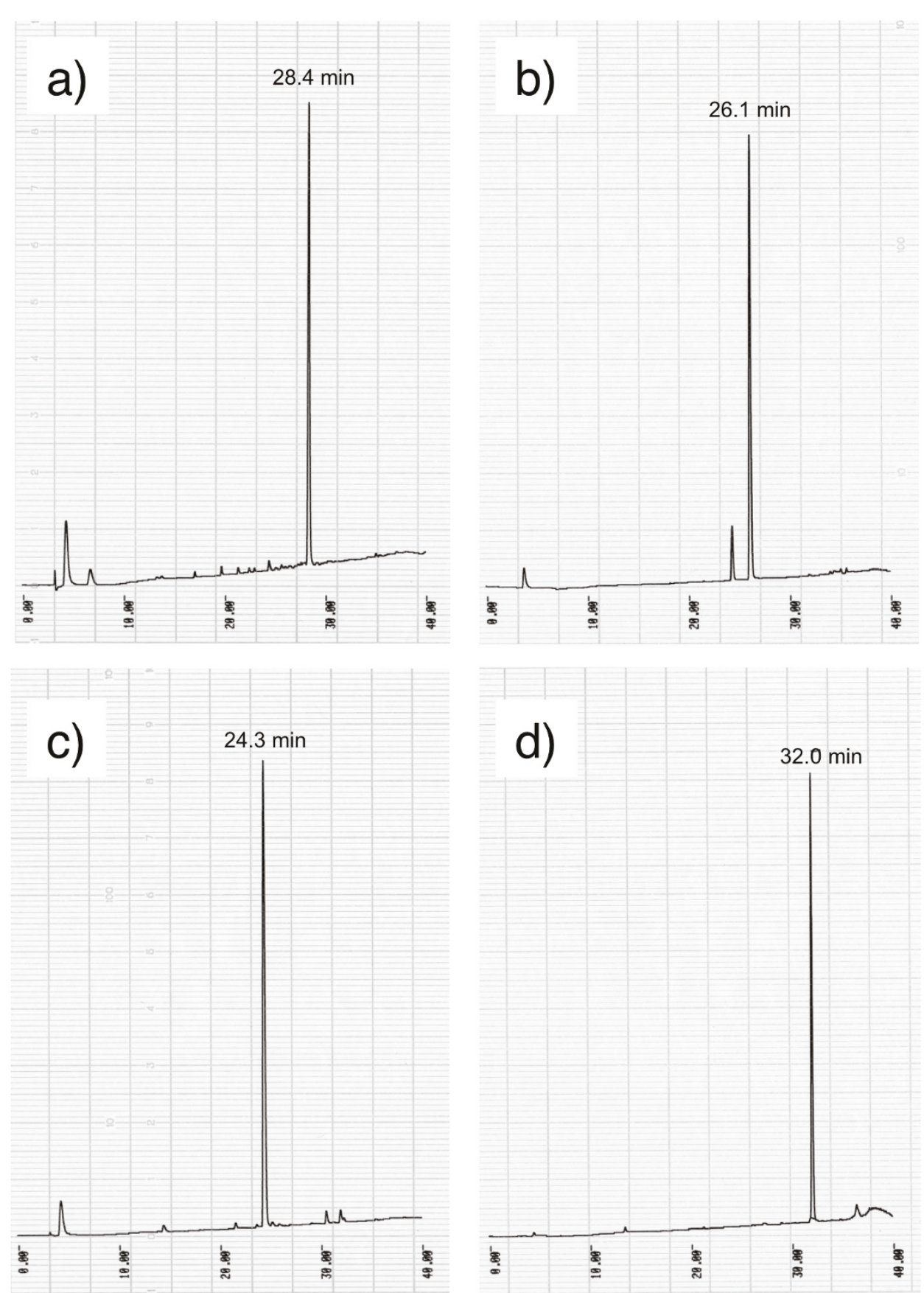

Figure 11 Analytical HPLC profiles of peptides obtained by in-water synthesis. a) Boc- Phe-Leu-NH2. b) Boc-Gly-Phe-Leu-NH2. c) Boc-GlyGly-Phe-Leu- $\mathrm{NH}_{2}$. d) Boc-Tyr(tBu)-Gly-Gly-Phe-Leu- $\mathrm{NH}_{2}$. Elution was carried out for over $30 \mathrm{~min}$ at a flow rate of $1 \mathrm{ml} / \mathrm{min}$ with a linear gradient from 9:1 to $3: 7$ mixture of $0.05 \%$ aqueous TFA and $0.05 \%$ TFA in acetonitrile.

produced in high purity $(\geq 90 \%)$ and high yield $(\geq 80 \%)$, indicating that the condensation reaction with waterdispersible nanoparticles proceeded smoothly in water (Table 5). The synthetic yield of Leu-enkephalinamide obtained by final deprotection was also high (51\%). Hydrophobic molecules cluster together in water. If there are nanosized reaction fields that help molecules to cluster more easily, reactions will proceed more efficiently. For reactions in water, it is also necessary to increase the affinity of the reaction fields for water. If water-dispersible nanoparticles that are poorly soluble in water are used, they may disperse uniformly in water, provide awareness between 
Table 5 Yield and purity of in-water synthetic peptides ${ }^{a}$

\begin{tabular}{|c|c|c|}
\hline Synthetic Peptide & Yield [\%] $^{b}$ & Purity [\%] $^{b}$ \\
\hline Boc-Phe-Leu-NH ${ }_{2}$ & 89 & 90 \\
\hline Boc-Gly-Phe-Leu-NH ${ }_{2}$ & 82 & 90 \\
\hline Boc-Gly-Gly-Phe-Leu-NH 2 & 84 & 92 \\
\hline Boc-Tyr(tBu)-Gly-Gly-Phe-Leu-NH${ }_{2}$ & 86 & 93 \\
\hline
\end{tabular}

${ }^{a}$ In-water coupling reactions were carried out with DMTMM. Purity of peptides was calculated from analytical HPLC profiles. ${ }^{b}$ Yield and purity of peptides was calculated from analytical HPLC profiles.

molecules by means of their hydrophobicity, function as water-friendly nanosized reaction fields, and thereby achieve a high yield. Nanoparticles are much smaller than ordinary particles and have very large specific surface areas. Thus, nanoparticles have new physical properties and are expected to produce new reactions.

This method is superior to conventional methods in terms of cost effectiveness, resource conservation, and environment friendliness. Because, after a reaction, synthetic protected peptides precipitate and can be isolated easily by filtration. That is, the use of this method will simplify manufacturing processes.

\section{Conclusion}

Consumption of organic solvents can be radically reduced by developing alternative methods that utilize water. There are obviously many potential advantages in replacing the traditional organic solvent-based methods with the water in synthetic chemistry. Recently, many new approaches are being developed, including a catalytic reaction process in water. Many of these new approaches, however, are limited to fields of research in laboratories, and there are a few applications to industrial processes, because only water-soluble materials can be used for them. In general, many organic compounds of synthetic intermediates for industrial use are poorly soluble in water, and therefore inadequate for reactions in water. Establishment of a technique that effectively accelerates reactions in water will make it possible to use water in industrial processes in many chemical synthesis plants, including those for compounds poorly soluble in water. This is the first technique reported that focuses on the conversion of insoluble reacting molecules themselves to water-dispersible nanoparticles. Control on the production of chemical substances, including the establishment of emission regulations, has been strengthened more than ever for environmental protection on a global scale. We hope that development of this technology will contribute to the construction of a sustainable industrial structure.

\section{Acknowledgements}

This work was supported in part by a Grant-in-Aid for Scientific Research and by an "Academic Frontier" Project for Private Universities: matching fund subsidy from the Japanese Ministry of Education, Culture, Sports, Science and Technology, 2006-2010.

\section{Authors' contributions}

$\mathrm{KH}$ wrote the manuscript. $\mathrm{KH}$ supervised the project. All authors discussed the results and commented on the manuscript.

\section{Competing interests}

The authors declare that they have no competing interests.

Received: 6 June 2011 Accepted: 25 August 2011

Published: 25 August 2011

References

1. Anastas PT, Warner JC: Green Chemistry: Theory and Practice New York, NY: Oxford University Press; 1998.

2. Winterton N: Twelve more green chemistry principles. Green Chem 2001, 3:G73-G75.

3. Sheldon RA: The E Factor: fifteen years on. Green Chem 2007, 9:1273-1283.

4. Hojo K, Maeda M, Kawasaki K: Solid-phase peptide synthesis in water. III. A water-soluble $\mathrm{N}$-protecting group, 2-[phenyl(methyl)sulfonio] ethoxycarbonyl tetrafluoroborate, and its application to peptide synthesis. Tetrahedron 2004, 60:1875-1866.

5. Hojo K, Maeda M, Kawasaki K: 2-[Phenyl(methyl)sulfonio]ethoxycarbonyl tetrafluoroborate, and its application to peptide synthesis. J Peptide Sci 2001, 7:615-618.

6. Hojo K, Maeda M, Kita E, Yamamoto S, Yamaguchi F, Kawasaki K: Peptide synthesis in water IV. Preparation of $\mathrm{N}$-ethanesulfonylethoxycarbonyl (Esc) amino Acids and their application to solid phase peptide synthesis. Chem Pharm Bull 2001, 52:422-427.

7. Hojo K, Maeda M, Kawasaki K: 2-(4-Sulfophenyl)ethoxycarbonyl group: a new water-soluble $N$-protecting group and its application to solid-phase peptide synthesis in water. Tetrahedron Lett 2004, 45:9293-9295.

8. McKay FC, Albertson WF: New Amine-masking Groups for Peptide Synthesis. J Am Chem Soc 1957, 79:4686-4690.

9. Carpino LA, Han G: The 9-fluorenylmethoxycarbonyl function, a new basesensitive. amino-protecting group. J Am Chem Soc 1970, 92:5748-5749.

10. Hojo K, Ichikawa H, Maeda M, Kida S, Fukumori Y, Kawasaki K: Solid-phase peptide synthesis using nanoparticulate amino acids in water. $J$ Peptide Sci 2007, 13:493-497.

11. Hojo K, Ichikawa H, Fukumori Y, Kawasaki K: Development of a method for solid-phase peptide synthesis in water. Int J Peptide Res Ther 2008, 14:373-380.

12. Hojo K, Mare Onishi, Ichikawa H, Fukumori Y, Kawasaki K: Peptide synthesis "in water" by a solution-phase method using water-dispersible nanoparticle Boc-amino acid. J Peptide Sci 2011, 17:487-492.

13. Merrifield RB: Solid-Phase Peptide Synthesis. 1. The synthesis of a tetrapeptide. J Am Chem Soc J Am Chem Soc 1963, 85:2149-2154.

14. Rabinow BE: Nanosuspensions in drug delivery. Nat Rev Discov 2004 3:785-795

15. Liversidge GG, Conzentino P: Drug particle size reduction for decreasing gastric irritancy and enhancing absorption of. naproxen in rats. Int J Pharm Sci 1995, 125:309-313.

16. Liversidge GG, Cundy KC: Particle size reduction for improvement of oral bioavailability of hydrophobic drugs: I. Absolute oral bioavailability of nanocrystalline danazol in beagle dogs. Int J Pharm Sci 1995, 125:91-97.

17. Sheehan JC, Hlavka JJ: The use of water-soluble and basic carbodiimides in peptide synthesis. J Org Chem 1956, 21:439-441.

18. Fujino M, Kobayashi S, Obayashi M, Fukuda T, Shinagawa S, Nishimura O: The use of $\mathrm{N}$-hydroxy-5-norbornene-2,3-dicarboximide active esters in peptide synthesis. Chem Pharm Bull 1974, 22:1857-1863.

19. Kaminski ZJ, Paneth P, Rudzinski JA: Study on the activation of carboxylic acids by means of 2-chloro-4,6-dimethoxy-1,3,5-triazine and 2-chloro4,6-diphenoxy-1.3.5-triazine. J Org Chem 1998, 63:4248-4225.

20. Kunishima M, Kawachi C, Morita J, Terao K, lawasaki F, Tani S: 4-(4,6Dimethoxy-1,3,5-triazin-2-yl)-4-methyl-morpholinium chloride: an efficient condensing agent leading to the formulation of amide and esters. Tetrahedron 1996, 55:13159-13179.

21. Anderson GW, Zimmerman JE, Callahan FM: The use of esters of $N$ hydroxysuccinimide in peptide synthesis. J Am Chem Soc 1964, 86:1839-1842.

22. Zimmerman JE, Anderson GW: The effect of active ester components on recemization in the synhesis of peptides by the dicyclohexylcarbodiimide method. J Am Chem Soc 1967, 89:7151-7152. 
23. Staros JV, Wright RW, Swingle DM: Enhancement by Nhydroxysulfosuccinimide of water-solublecarbodiimide-mediated coupling reactions. Anal Biochem 1986, 156:220-222.

doi:10.1186/1752-153X-5-49

Cite this article as: Hojo et al:: Development of a method for environmentally friendly chemical peptide synthesis in water using water-dispersible amino acid nanoparticles. Chemistry Central Journal 2011 5:49.

\footnotetext{
Publish with ChemistryCentral and every scientist can read your work free of charge

"Open access provides opportunities to our colleagues in other parts of the globe, by allowing anyone to view the content free of charge."

W. Jeffery Hurst, The Hershey Company.

- available free of charge to the entire scientific community

- peer reviewed and published immediately upon acceptance

- cited in PubMed and archived on PubMed Central

- yours - you keep the copyright

Submit your manuscript here:

hubmit your manuscript here:
http://www.chemistrycentral.com/manuscript/<smiles>c1ccccc1</smiles> 